\title{
Singlet exciton condensation and bond-order-wave phase in the extended Hubbard model
}

\author{
Mohsen Hafez-Torbati ${ }^{1, *}$ and Götz S. Uhrig ${ }^{1, \dagger}$ \\ ${ }^{1}$ Lehrstuhl für Theoretische Physik I, Technische Universität Dortmund, \\ Otto-Hahn-Straße 4, 44221 Dortmund, Germany
}

(Dated: September 27, 2017)

\begin{abstract}
The competition of interactions implies the compensation of standard mechanisms which leads to the emergence of exotic phases between conventional phases. The extended Hubbard model (EHM) is a fundamental example for the competition of the local Hubbard interaction and the nearest-neighbor density-density interaction, which at half-filling and in one dimension leads to a bond order wave (BOW) between a charge density wave (CDW) and a quasi-long-range order Mott insulator (MI). We study the full momentum-resolved excitation spectrum of the one dimensional EHM in the CDW phase and clarify the relation between different elementary energy gaps. We show that the CDW-to-BOW transition is driven by the softening of a singlet exciton at momentum $\pi$. The BOW is realized as the condensate of this singlet exciton.
\end{abstract}

PACS numbers: $71.30 .+\mathrm{h}, 71.10 \cdot \mathrm{Li}, 71 \cdot 10 . \mathrm{Fd}, 74.20 . \mathrm{Fg}$

\section{INTRODUCTION}

Strong interactions among electrons can lead to the emergence of collective phenomena such as the stabilization of new phases of matter which host non-trivial elementary excitations ${ }^{1,2}$. The role of onsite Hubbard interaction and its competition with different kinetic terms is widely investigated ${ }^{3-9}$. However, relatively less attention is paid to the effect of non-local short-range interactions such as first or second neighbor density-density interaction terms. The possible spontaneous emergence of quantum anomalous Hall state (for spinless case) and quantum spin Hall state (for spinfull case) on the honeycomb lattice due to first and the second neighbor interactions serves as an interesting controversial example in the field of topological Mott insulator ${ }^{10-12}$.

In order to study quantum phase transitions and to search for non-trivial quantum states it is wellestablished to analyze effective models with competing interaction terms. Their competition compensates the driving mechanisms of rather trivial phases so that the non-compensated higher order terms dominate the physics ${ }^{13-15}$. An example is the extended Hubbard model (EHM) at half filling where two interactions, namely the onsite Hubbard repulsion $U$ and the nearest-neighbor (NN) repulsion $V$ compete.

We study the half-filled EHM in one dimension at zero temperature; its Hamiltonian reads

$$
\begin{aligned}
H= & t \sum_{i \sigma}\left(c_{i, \sigma}^{\dagger} c_{i+1, \sigma}+\text { h.c. }\right)+V \sum_{i}\left(n_{i}-1\right)\left(n_{i+1}-1\right) \\
& +U \sum_{i}\left(n_{i, \uparrow}-1 / 2\right)\left(n_{i, \downarrow}-1 / 2\right)
\end{aligned}
$$

where $c_{i, \sigma}$ and $c_{i, \sigma}^{\dagger}$ are electron annihilation and creation operators at site $i$ with spin $\sigma$, respectively. The density operator $n_{i, \sigma}:=c_{i, \sigma}^{\dagger} c_{i, \sigma}$ counts the number of electrons with spin $\sigma$ at site $i$ and $n_{i}:=n_{i, \uparrow}+n_{i, \downarrow}$. In the twofold degenerate CDW regime $(V \gg U)$, the gaps to both

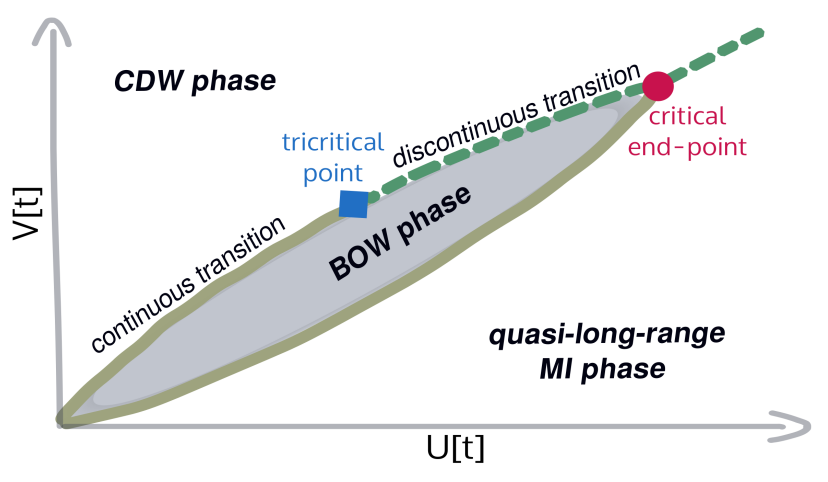

Figure 1. (Color online) Schematic phase diagram of the extended Hubbard model (1) found by bosonization ${ }^{16-20}$, quantum Monte $\mathrm{Carlo}^{21-23}$, and density matrix renormalization $\operatorname{group~method~}^{24-26}$.

singlet and triplet excitations are finite. In the MI $(U \gg$ $V)$, the charge degrees of freedom are frozen and the low-energy physics is captured by the Heisenberg model with quasi long-range magnetic order and gapless spin excitations.

The phase diagram of the EHM (1), see Fig. 1, has been studied extensively using bosonization ${ }^{16,17}$, renormalization group ${ }^{18-20}$, quantum Monte Carlo (QMC) ${ }^{21-23}$, and density matrix renormalization group (DMRG) ${ }^{24-26}$ '. The CDW and the MI are separated by the intermediate BOW phase for small to intermediate values of $U$ and $V$. For large $U$ and $V$ values beyond a critical end point, the BOW disappears and a direct first order transition from the CDW to the MI is observed. The CDW-toBOW transition changes from second order to first order beyond a tricritical point while the BOW-to-MI transition remains second order. Modified models with similar tricritical points have also been studied ${ }^{27,28}$.

The phase transitions in the EHM (1) are determined by computing various correlation functions as well as charge and spin gaps. It is reported that at the second 
order CDW-to-BOW transition the charge gap vanishes while the spin gap remains finite ${ }^{26}$. In the MI, the spin gap is zero and holon-antiholon form bound states ${ }^{29}$. In previous investigations, however, the possible formation of an electron-hole bound state with $S=0$, i.e., of a singlet exciton, has not been considered.

In this paper, we present the full momentum-resolved low-energy spectrum of the EHM (1) in the CDW phase close to the transition using continuous unitary transformations (CUTs) ${ }^{30-32}$. A rich excitation spectrum comprising two singlet and two triplet bound states is identified. In contrast to the DMRG analysis ${ }^{26}$, we find that the second order transition from the CDW to the BOW is induced by the vanishing of the optical gap, i.e., the energy of a singlet exciton at total momentum $k=\pi$ vanishes. The bond order reflects the condensate of these singlet excitons and can be understood by a BCS-like mean-field theory. The spin gap remains finite and is smaller than the charge gap at the transition point.

\section{CHARGE DENSITY WAVE}

The ground state has total spin $S=0$. In order to track phase transitions we consider four excitation gaps: the 1-particle gap $\Delta_{1}$, the charge gap $\Delta_{c}$, the singlet exciton gap (or optical gap) $\Delta_{e}$, and the spin gap $\Delta_{s}$ defined by ${ }^{33}$

$$
\begin{aligned}
\Delta_{1} & :=E_{0}^{L+1}+E_{0}^{L-1}-2 E_{0}^{L}=2\left(E_{0}^{L+1}-E_{0}^{L}\right) \\
\Delta_{c} & :=\frac{1}{2}\left(E_{0}^{L+2}+E_{0}^{L-2}-2 E_{0}^{L}\right)=E_{0}^{L+2}-E_{0}^{L} \\
\Delta_{e} & :=E_{1, S=0}^{L}-E_{0}^{L} \\
\Delta_{s} & :=E_{1, S=1}^{L}-E_{0}^{L}
\end{aligned}
$$

where $E_{0}^{N}$ is the ground state energy at $N$ electrons; at half-filling $N=L$ holds where $L$ is the number of lattice sites. The energy $E_{1, S}^{N}$ corresponds to the first excited state with total spin $S$ and $N$ electrons. The 1particle gap measures the minimum energy required for adding a single electron and a single hole to the system. The charge gap $\Delta_{c}$ lies below the 1-particle $\Delta_{1}$ gap only in the case of Cooper-pair formation otherwise they are equal. The second equalities in (2a) and (2b) hold due to particle-hole symmetry. An electron-hole pair can form a bound state (exciton) in the singlet and/or in the triplet channel. Its energy defines the singlet and the spin gap, respectively. We stress that this consideration implies that the singlet and the spin gap must be equal or smaller than the 1-particle gap. If the gaps are smaller the difference in energy is the excitonic binding energy. We notice that a charge gap smaller than the spin gap as suggested in DMRG analysis ${ }^{26}$ can only be understood based on electron-electron (hole-hole) bound states.

Different definitions are used for the charge gap in different contexts and we have to clarify this point before proceeding. The singlet exciton gap (2c) and the spin gap (2d) can be extracted from the Fourier transform of the charge-charge $\left\langle n_{i} n_{i+d}\right\rangle$ and the spin-spin $\left\langle S_{i}^{z} S_{i+d}^{z}\right\rangle$ correlation functions, respectively, as calculated for the 1D EHM by QMC method in Refs. 22 and 23. What is called "charge gap" in these references is equivalent to our singlet exciton gap Eq. (2c). The singlet exciton gap and the spin gap are also the gaps addressed in bosonization ${ }^{16-19}$ as the bosonized field always create pair of electron and hole. We notice that for the proper treatment of the 1-particle gap (2a) and the charge gap (2b) in the bosonization approach the explicit consideration of Klein factors would be necessary ${ }^{34}$.

In the atomic limit $(t=0)$ and for $2 V>U$ the ground state is a two-fold degenerate CDW where empty and fully occupied sites alternate, see Figs. 2a.1 and 2a.2. The system becomes excited if an electron hops from an occupied site to an empty one creating an electron-hole pair in Figs. 2a.3 and 2a.4. The 1-particle gap is given by $\Delta_{1}=4 V-U$ as can be read off from Fig. 2a.3 where electron and hole are separated. To minimize its energy, the electron-hole pair can form a bound state on NN sites so that the singlet (and the spin gap) is given by $\Delta_{s}=\Delta_{e}=3 V-U$, see Fig. 2a.4. A single domain-wall separating the two degenerate ground states is depicted in Fig. 2a.5, requiring the excitation energy $2 \mathrm{~V}-U / 2$, i.e., $\Delta_{1} / 2$.

From this simple argument, one can deduce that the $\mathrm{NN}$ interaction strongly favors the formation of neutral exciton. The degeneracy of the singlet and the triplet gap in the atomic limit is lifted due to NN hopping. We show that these bound states survive even close to the CDW-to-BOW transition. A similar scenario of exciton formation due to NN interaction has been found in related models ${ }^{35,36}$.

We take the CDW in Fig. 2a.1 as reference state. The electron-hole transformation $T^{(\mathrm{e}-\mathrm{h})}: c_{i, \sigma}^{\dagger} \rightarrow h_{i, \sigma}$ on the odd sublattice expresses the EHM (1) in terms of quasiparticles (QPs). This means that any creation operator after the transformation stands for the creation of an excitation: adding an electron to an empty site or adding a hole to an fully occupied site. Then, the electron and hole operators are uniformly denoted by the fermion operator $f_{i, \sigma}^{(\dagger)}$. After the local transformation $T^{(1)}: f_{j, \sigma}^{\dagger} \rightarrow e^{i \frac{\pi}{2} j} e^{-i \frac{\pi}{4}} f_{j, \sigma}^{\dagger}$ the EHM can be written as

$$
\begin{aligned}
H= & \frac{U-4 V}{4} \sum_{i} \mathbb{1}+\frac{4 V-U}{2} \sum_{i, \sigma} f_{i, \sigma}^{\dagger} f_{i, \sigma} \\
& +U \sum_{i} f_{i, \uparrow}^{\dagger} f_{i, \uparrow} f_{i, \downarrow}^{\dagger} f_{i, \downarrow}-V \sum_{i \sigma \beta} f_{i, \sigma}^{\dagger} f_{i, \sigma} f_{i+1, \beta}^{\dagger} f_{i+1, \beta} \\
& +t \sum_{i, \sigma}\left(f_{i, \sigma}^{\dagger} f_{i+1, \sigma}^{\dagger}+\text { h.c. }\right) .
\end{aligned}
$$

The local transformation $T^{(1)}$ has restored the full translational symmetry facilitating the subsequent analysis. In the QP representation, the original hopping term has become a Bogoliubov term creating a singlet pair of fermions on NN sites. We stress that the NN electron- 
(a)

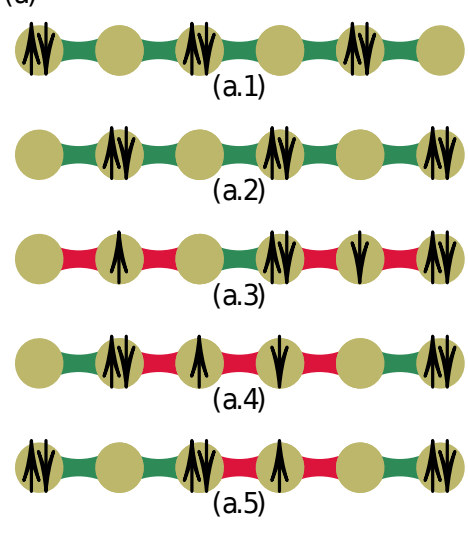

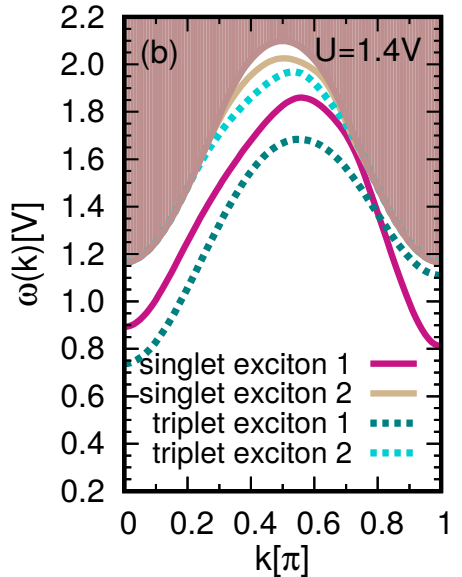

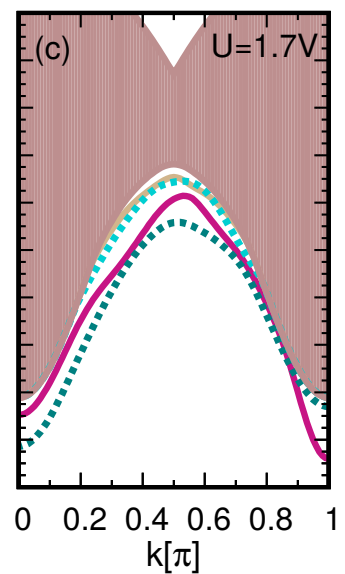

Figure 2. (Color online) (a) Schematic representation of the two degenerate CDWs (a.1 and a.2), of an excited electron-hole pair (a.3 and a.4), and of a single domain-wall (a.5) on a piece of chain of six sites. The bonds low in energy due to $V$ in (1) are shown in green, the ones high in energy are shown in red. Clearly, configurations where electron and hole are close together are favored suggesting binding. Excitation spectrum of the EHM (1) in the CDW for $U=1.4 \mathrm{~V}$ (b) and $U=1.7 \mathrm{~V}$ (c). (d) The 1-particle gap $\Delta_{1}$, singlet gap $\Delta_{e}$, and spin gap $\Delta_{s}$, defined in (2), vs. the onsite interaction $U$. The hopping $t$ is set to $0.5 V$ and the order of the deepCUT is 10 .

electron interaction in (3) has acquired a minus sign indicating attraction between the original electron and hole.

To eliminate the Bogoliubov terms which change the number of QPs we employ the directly evaluated enhanced perturbative CUT (deepCUT) ${ }^{37}$. The resulting effective model allows us to analyze the complete momentum-resolved excitation spectrum of the Hamiltonian (3). The CUT is performed in the thermodynamic limit and is known as a powerful approach to compute excitation spectra and spectral densities ${ }^{38-41}$. We treat the Bogoliubov term as the perturbation in the deepCUT formalism $^{37}$ so that the flow equations are truncated in powers of the hopping $t$. The same symmetries and simplification rules can be used as in Ref. 40.

In the CUT method, the Hamiltonian is mapped to an effective one by a unitary transformation which depends on an auxiliary parameter $\ell$. The transformed Hamiltonian satisfies the flow equation ${ }^{30-32}$

$$
\partial_{\ell} H(\ell)=[\eta(\ell), H(\ell)]
$$

where the antihermitian operator $\eta(\ell)$ is the generator of the flow and determines the essence of the transformation. We decompose the Hamiltonian into different parts which create and annihilate specific numbers of $\mathrm{QPs}^{31,37}$ :

$$
H(\ell)=\sum_{n, m} H_{n: m}(\ell)
$$

where $H_{n: m}$ creates $n$ and annihilates $m$ QPs. The reduced generator ${ }^{42}$

$$
\eta_{p: x}=\sum_{m=0}^{x} \sum_{n>m}\left(H_{n: m}-\text { h.c. }\right)
$$

allows us to decouple the first $x$ QP sectors from higher sectors.
Using the reduced generator $\eta_{p: 2}$ to decouple up to two QPs sector in the EHM leads to a diverging flow because the decoupling of the subspaces with two QPs is difficult if binding phenomena prevail. Hence we used the $\eta_{p: 1}$ generator instead and implemented the diagonalization in the 2-QP subspace ${ }^{40}$. This means that the off-diagonal terms linking the 2-QP sector to 4 and higher QP sectors are neglected. This procedure can be understood as a variational approximation for the effective Hamiltonian derived from deepCUT. The neglected terms would only increase the binding energies, thereby enhancing the effects discussed in this work. Moreover, we know from data in order 6 close to the transition where the $\eta_{p: 2}$ generator still converges, that the obtained results are quantitatively close to the ones obtained using the $\eta_{p: 1}$ generator so that we conclude that the neglected off-diagonal elements are of minor importance.

In Figs. $2 \mathrm{~b}(U=1.4 \mathrm{~V})$ and $2 \mathrm{c}(U=1.7 \mathrm{~V})$ the excitation spectrum of the EHM (1) is depicted in the CDW phase. The neutral singlet (triplet) excitons are specified by solid (dashed) lines. The solid areas indicate the electron-hole continua constructed from the single fermion dispersion. A rich excitation spectrum comprising two singlet and two triplet neutral excitons is identified. We have not found any electron-electron (hole-hole) bound state in the entire Brillouin zone. The exciton 1 exists almost in the whole Brillouin zone while the exciton 2 is present only close to $k=\pi / 2$ (lattice constant is set to unity). We ascribe the small wiggles close to $k=\pi / 2$ to the truncation in finite order. For $U=1.4 \mathrm{~V}$, the singlet exciton 1 takes its minimum energy at $k=\pi$. This minimum is higher in energy than the minimum of the triplet exciton 1 at $k=0$. Increasing, however, the Hubbard interaction to $U=1.7 \mathrm{~V}$ one discerns in Fig. $2 \mathrm{c}$ that the lowest excited state is the singlet exciton 1 
at $k=\pi$. It is this singlet exciton which becomes softs at the transition to the BOW upon increasing $U$ further. Beyond the transition it forms a macroscopic condensate, i.e., the BOW. The same behavior is found in order 6 and 8. This is consistent with bosonization ${ }^{16-19}$ and QMC analysis $^{22,23}$ which suggest vanishing of a neutral spinless gap at the CDW-to-BOW transition, but disagrees with $\mathrm{DMRG}^{26}$ which proposes the vanishing of the charge gap $(2 \mathrm{~b})$.

According to the definitions in (2), the 1-particle gap is given by the lowest energy of the electron-hole continuum which occurs at $k=0$ and $k=\pi$. The charge gap equals the 1-particle gap as no electron-electron bound state is found. The singlet exciton 1 at $k=\pi$ and the triplet exciton 1 at $k=0$ define the singlet and the spin gap, respectively. This clarifies the difference between the 1particle gap $\Delta_{1}$ and the singlet gap $\Delta_{e}$. The dependences of the gaps on $U$ is presented in Fig. 2 d. For $U \lesssim 1.57 \mathrm{~V}$ the lowest excitation has $S=1$ while for $U \gtrsim 1.57 \mathrm{~V}$ it has $S=0$. The singlet gap vanishes at the transition $U_{c 1} \simeq 1.91 \mathrm{~V}$ while the 1 -particle gap remains finite and larger than the spin gap. This modifies the currently used scenario where the charge gap is zero at finite spin gap at the CDW-to-BOW transition ${ }^{26}$. Note that the results in Fig. 2d are valid only up to the transition.

\section{SINGLET EXCITON CONDENSATION AND BOND ORDER WAVE}

Once the energy of an exciton falls below zero its creation lowers the total energy of the system. Hence, more and more of them will be created leading to a macroscopic occupation: a condensate is formed. This continued exciton creation comes to an end due to residual repulsive interactions between them. Such interactions exist because only the composite object, the exciton, behaves like a boson. The internal fermionic structure prevents two excitons to come too close.

This physics is captured by a BCS-type mean-field theory applied to the effective Hamiltonian systematically derived by deepCUT beyond the CDW-to-BOW transition at $U=U_{c 1}$. Here, we show in this way that the condensation of the singlet exciton at $k=\pi$ leads to the BOW. Of course, the critical fluctuations of the transition and thus its critical exponents are not accounted for by the BCS theory, but our focus is here on the driving mechanisms resulting from the fundamental energies in the system. Critical behavior may be captured by bosonization $^{16,17}$, analytical ${ }^{18,20}$ or numerical ${ }^{24-26}$ renormalization approaches or quantum Monte Carlo ${ }^{22,23}$.

To describe the BOW, we consider the effective Hamiltonian from the deepCUT up to quartic level

$$
H_{\mathrm{eff}}=E_{0}+\sum_{i j} \Gamma_{j ; i} f_{j}^{\dagger} f_{i}+\sum_{k l i j} \Gamma_{k l ; i j} f_{l}^{\dagger} f_{k}^{\dagger} f_{i} f_{j},
$$

where the range of hopping and interaction processes in (7) is limited by the order of the truncation. The quartic Hamiltonian (7) captures the condensation of 2$\mathrm{QP}$ bound states. In the BCS analysis, we allow for finite expectation values $\left\langle f_{i, \sigma}^{\dagger} f_{i+m, \sigma}^{\dagger}\right\rangle$ and $\left\langle f_{i, \sigma}^{\dagger} f_{i+n, \sigma}\right\rangle$ where $m$ and $n$ are restricted to odd and even numbers, respectively, due to the conservation of the total charge. We also allow for broken translational symme$\operatorname{try}\left\langle f_{i, \sigma}^{\dagger} f_{i+m, \sigma}^{\dagger}\right\rangle \neq\left\langle f_{i+1, \sigma}^{\dagger} f_{i+m+1, \sigma}^{\dagger}\right\rangle$ to account for the possibility of a BOW. ${ }^{40}$

The bilinear Hamiltonian resulting from the application of Wick theorem on $(7)$ reads $^{40}$

$$
\begin{aligned}
H & =\tilde{\mathrm{E}}_{0}+\sum_{r \sigma} \sum_{m} \Delta_{m}^{r}\left(: f_{r, \sigma}^{\dagger} f_{r+m, \sigma}^{\dagger}:+ \text { h.c. }\right) \\
& +\sum_{r \sigma}\left(t_{0}: f_{r, \sigma}^{\dagger} f_{r, \sigma}:+\sum_{n} t_{n}\left(: f_{r, \sigma}^{\dagger} f_{r+n, \sigma}:+ \text { h.c. }\right)\right)(8)
\end{aligned}
$$

where the Bogoliubov prefactor $\Delta_{m}^{r}$ changes from odd to even sublattice. We consider $\Delta_{m}^{r}=\Delta_{m}^{A}$ for $r$ even and $\Delta_{m}^{r}=\Delta_{m}^{B}$ for $r$ odd. The prefactors $\tilde{\mathrm{E}}_{0}, t_{n}, \Delta_{m}^{A}$, and $\Delta_{m}^{B}$ depend on the coefficients of the effective Hamiltonian (7) and the bilinear expectation values which are to be determined self-consistently. The BCS Hamiltonian (8) is diagonalized in momentum space by a Bogoliubov transformation. After some standard calculations one obtains the self-consistent equations

$$
\begin{aligned}
&\left\langle f_{r, \sigma}^{\dagger} f_{r+n, \sigma}\right\rangle= \frac{1}{\pi} \int_{0}^{\frac{\pi}{2}} d k \frac{\lambda(k)-t(k)}{\lambda(k)} \cos (n k), \\
&\left\langle f_{r, \sigma}^{\dagger} f_{r+m, \sigma}^{\dagger}\right\rangle=\frac{1}{\pi} \int_{0}^{\frac{\pi}{2}} \frac{d k}{\lambda(k)}(\operatorname{Im}(\Delta(k)) \sin (m k) \\
&\left.-(-1)^{r} \operatorname{Re}(\Delta(k)) \cos (m k)\right) .
\end{aligned}
$$

We have defined the functions $t(k), \Delta(k)$, and $\lambda(k)$ as

$$
\begin{aligned}
t(k) & =t_{0}+2 \sum_{n} t_{n} \cos (n k) \\
\Delta(k) & =\sum_{m}\left(\left(\Delta_{m}^{A}-\Delta_{m}^{B}\right) \cos (m k)-i\left(\Delta_{m}^{A}+\Delta_{m}^{B}\right) \sin (m k)\right) \\
\lambda(k) & =\sqrt{t^{2}(k)+|\Delta(k)|^{2}},
\end{aligned}
$$

where $n$ and $m$ take positive even and positive odd values, respectively.

The BCS analysis is exact in the entire CDW phase where the quantum fluctuations are already captured by the deepCUT. In the condensate phase, i.e., beyond $U_{c 1}$, it is an approximation as mentioned above. The energy differences are rendered quite reliably as long as the system is not shifted too far beyond the transition.

For $U<U_{c 1}$, all expectation values are trivially zero because the deepCUT has mapped the ground state of the EHM (1) to the vacuum of QPs. Beyond $U_{c 1}$, the expectation values become finite. Two degenerate solutions I and II are found corresponding to the two ways to break the translational symmetry by bond order. We 

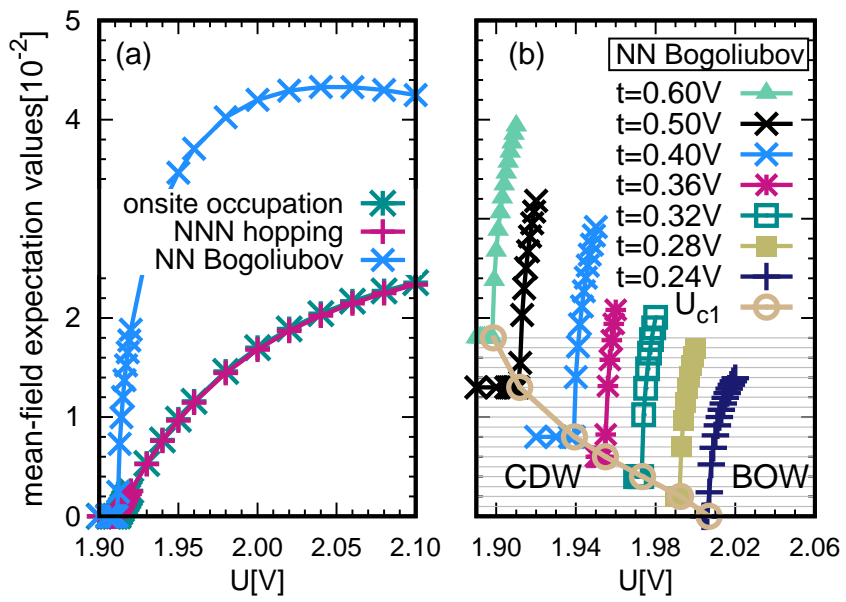

Figure 3. (Color online) (a) The onsite occupation $\left\langle f_{i, \sigma}^{\dagger} f_{i, \sigma}\right\rangle$, the NN Bogoliubov term $\left\langle f_{i, \sigma}^{\dagger} f_{i+1, \sigma}^{\dagger}\right\rangle$, and the next-nearestneighbor (NNN) hopping $\left\langle f_{i, \sigma}^{\dagger} f_{i+2, \sigma}\right\rangle$ vs. $U$ for $t=0.5 \mathrm{~V}$. (b) The NN Bogoliubov term shifted by $(t-0.24) \times 0.05$ along the $y$-axis for clarity at various values of the hopping $t$ as function of $U$. The order of the underlying deepCUT is 10 .

obtain $\left\langle f_{i, \sigma}^{\dagger} f_{i+m, \sigma}^{\dagger}\right\rangle=-\left\langle f_{i+1, \sigma}^{\dagger} f_{i+m+1, \sigma}^{\dagger}\right\rangle$. The two solutions are related via $\left\langle f_{i, \sigma}^{\dagger} f_{i+m, \sigma}^{\dagger}\right\rangle_{I}=-\left\langle f_{i, \sigma}^{\dagger} f_{i+m, \sigma}^{\dagger}\right\rangle_{I I}$. The natural order parameter of the BOW is the difference of the expectation values on adjacent NN bonds.

In Fig. 3a we depict the important expectation values of the BCS mean-field solution as function of $U$. We stress that the value of $U$ where the expectation values become finite matches precisely the value where $\Delta_{e}$ hits zero in Fig. 2d. The NN Bogoliubov expectation value displays a square root behavior as usual in mean-field.

In Fig. 3b, the NN Bogoliubov term is plotted for various hopping parameters versus the Hubbard interaction $U$. From this figure, one can read off the transition line CDW-to-BOW phases, $U_{c 1}(t)$. We expect the CDW-toBOW transition to change from second order to first order below $t \simeq 0.32 \mathrm{~V}$ based on previous results ${ }^{23,26}$. From Fig. $3 \mathrm{~b}$, we find a second order transition at least down to $t=0.24 \mathrm{~V}$. Below $t=0.24 \mathrm{~V}$ even the reduced generator $n: 1$ diverges. The fact that we can not identify the tricritical point where the character of the transition changes may either result from the truncation of the effective Hamiltonian (7) to quartic terms or from the approximate treatment on mean-field level. Recall that finding first order transitions in Landau theory generically requires the inclusion of hexatic terms.

Furthermore, it has been proposed by Hirsch that the formation of MI "droplets" beyond a critical size in the CDW phase is responsible for the first order transition in the $\mathrm{EHM}^{21}$. If this is the mechanism of the first order transition one has to address multi-particle bound states which is beyond the scope of the present article. The proper description of multi-particle bound states requires to go beyond quartic level in (7) because the irreducible interactions of more than two QPs matter.

\section{CONCLUSIONS AND OUTLOOK}

Understanding unusual phases occurring between two more standard phases is currently a very active topic. A nice example are the phases occurring in fermionic lattice models such as the ionic Hubbard model or the extended Hubbard model. In the latter, the two interactions, the onsite one and the nearest-neighbor one, are competing. Where they compensate in one dimension neither the Mott insulator (MI) not the alternating charge density wave (CDW) occurs, but an alternating bond order wave (BOW).

In the present work, we have shown that the occurrence of the BOW can be understood from the CDW as the softening of a singlet exciton at momentum $k=\pi$. Thus, the bound state of an electron-hole pair represents an essential collective mode. Upon passing from the CDW to the BOW, for instance by increasing $U$, this mode condenses. Since the mode lives at $\pi$ its condensate naturally displays an alternating order. It is not accompanied by magnetic order because the condensing mode does not carry any spin. The same scenario occurred in the ionic Hubbard model ${ }^{40}$.

Our finding naturally implies that the singlet exciton gap $\Delta_{e}$ is smaller than the 1-particle gap $\Delta_{1}$ which reflects the energy needed to create an electron and a hole excitation independently, i.e., at large distance. For the spin gap $\Delta_{s}$ the relation $\Delta_{s} \leq \Delta_{1}$ holds as well because the spin excitation also represents an exciton, but with $S=1$. The differences $\Delta_{1}-\Delta_{e}$ and $\Delta_{1}-\Delta_{s}$ are the binding energies of the $S=0$ and the $S=1$ exciton, respectively.

So far, we could not find the first order transition for larger interactions $U, V$ corresponding to smaller hopping $t$. But we presume that multi-particle terms need to be included to capture this feature.

A particularly intriguing challenge is to extend the presented analysis to the two-dimensional extended Hubbard model as the deepCUT method has no conceptual problem with dimension and the BCS-mean-field theory is expected to work better in higher dimension ${ }^{9}$. There, very little is known about intermediate phases because many theoretical tools do not work in higher dimensions or only at considerably larger efforts. But the analogy to the ionic Hubbard model suggests that a rich scenario of intermediate phases occurs, breaking first discrete and then continuous symmetries upon increasing the Hubbard interaction ${ }^{9}$. The possible spontaneous emergence of quantum anomalous Hall state and quantum spin Hall state on the honeycomb lattice due to competing first and second neighbor interactions is another currently controversial issue which calls for future studies. ${ }^{10-12,43,44}$

\section{ACKNOWLEDGMENTS}

We thank Satoshi Ejima, Fabian Essler, Holger Fehske, and Bruce Normand for useful discussions. 
REFERENCES

* mohsen.hafez@tu-dortmund.de; Current address: Institut für Theoretische Physik, Goethe-Universität, 60438 Frankfurt/Main, Germany

† goetz.uhrig@tu-dortmund.de

1 L. Balents, Nature 464, 199 (2010).

2 T. Senthil, A. Vishwanath, L. Balents, S. Sachdev, and M. P. A. Fisher, Science 303, 1490 (2004).

${ }^{3}$ C. N. Varney, C.-R. Lee, Z. J. Bai, S. Chiesa, M. Jarrell, and R. T. Scalettar, Phys. Rev. B 80, 075116 (2009).

${ }^{4}$ Z. Y. Meng, T. C. Lang, S. Wessel, F. F. Assaad, and A. Muramatsu, Nature 464, 847 (2010).

5 S. Sorella, Y. Otsuka, and S. Yunoki, 2, 992 (2012).

${ }^{6}$ H.-Y. Yang, A. M. Läuchli, F. Mila, and K. P. Schmidt, Phys. Rev. Lett. 105, 267204 (2010).

7 D. Cocks, P. P. Orth, S. Rachel, M. Buchhold, K. Le Hur, and W. Hofstetter, Phys. Rev. Lett. 109, 205303 (2012).

8 M. Ebrahimkhas, Z. Drezhegrighash, and E. Soltani, Physics Letters A 379, 1053 (2015).

9 M. Hafez-Torbati and G. S. Uhrig, Phys. Rev. B 93, 195128 (2016).

10 S. Raghu, X.-L. Qi, C. Honerkamp, and S.-C. Zhang, Phys. Rev. Lett. 100, 156401 (2008).

11 J. Motruk, A. G. Grushin, F. de Juan, and F. Pollmann, Phys. Rev. B 92, 085147 (2015).

12 D. D. Scherer, M. M. Scherer, and C. Honerkamp, Phys. Rev. B 92, 155137 (2015).

13 M. Fabrizio, A. O. Gogolin, and A. A. Nersesyan, Phys. Rev. Lett. 83, 2014 (1999).

14 L. Capriotti, F. Becca, A. Parola, and S. Sorella, Phys. Rev. Lett. 87, 097201 (2001).

15 A. W. Sandvik, Phys. Rev. Lett. 98, 227202 (2007).

${ }^{16}$ M. Nakamura, Journal of the Physical Society of Japan $\mathbf{6 8}$, 3123 (1999), http://dx.doi.org/10.1143/JPSJ.68.3123.

17 M. Nakamura, Phys. Rev. B 61, 16377 (2000).

18 M. Tsuchiizu and A. Furusaki, Phys. Rev. Lett. 88, 056402 (2002).

19 M. Tsuchiizu and A. Furusaki, Phys. Rev. B 69, 035103 (2004).

20 K.-M. Tam, S.-W. Tsai, and D. K. Campbell, Phys. Rev. Lett. 96, 036408 (2006).

21 J. E. Hirsch, Phys. Rev. Lett. 53, 2327 (1984).

${ }^{22}$ P. Sengupta, A. W. Sandvik, and D. K. Campbell, Phys. Rev. B 65, 155113 (2002).

23 A. W. Sandvik, L. Balents, and D. K. Campbell, Phys. Rev. Lett. 92, 236401 (2004).
${ }^{24}$ E. Jeckelmann, Phys. Rev. Lett. 89, 236401 (2002).

25 Y. Z. Zhang, Phys. Rev. Lett. 92, 246404 (2004).

26 S. Ejima and S. Nishimoto, Phys. Rev. Lett. 99, 216403 (2007).

27 F. Lange, S. Ejima, and H. Fehske, Phys. Rev. B 92, 041120 (2015)

28 S. Ejima, F. H. L. Essler, F. Lange, and H. Fehske, Phys. Rev. B 93, 235118 (2016).

29 F. H. L. Essler, F. Gebhard, and E. Jeckelmann, Phys. Rev. B 64, 125119 (2001).

30 F. Wegner, Annalen der Physik 506, 77 (1994).

31 C. Knetter and G. Uhrig, The European Physical Journal B - Condensed Matter and Complex Systems 13, 209 (2000).

32 S. Kehrein, The Flow Equation Approach to Many-Particle Systems, Springer Tracts in Modern Physics, Vol. 217 (Berlin:Springer, 2006).

33 In Ref. 39, we have considered the 1-particle gap, Eq. (2a), as charge gap. However, we notice that there is no difference between the charge gap and the 1-particle gap in the absence of Cooper-pair formation.

34 J. von Delft and H. Schoeller, Annalen der Physik 7, 225 (1998).

35 M. Hafez and S. A. Jafari, The European Physical Journal B 78, 323 (2010).

${ }^{36}$ M. Hafez and M. Abolhassani, Journal of Physics: Condensed Matter 23, 245602 (2011).

37 H. Krull, N. A. Drescher, and G. S. Uhrig, Phys. Rev. B 86, 125113 (2012).

38 C. Knetter, K. P. Schmidt, M. Grüninger, and G. S. Uhrig, Phys. Rev. Lett. 87, 167204 (2001).

39 C. Knetter and G. S. Uhrig, Phys. Rev. Lett. 92, 027204 (2004).

40 M. Hafez Torbati, N. A. Drescher, and G. S. Uhrig, Phys. Rev. B 89, 245126 (2014).

41 M. Powalski, G. S. Uhrig, and K. P. Schmidt, Phys. Rev. Lett. 115, 207202 (2015).

42 T. Fischer, S. Duffe, and G. S. Uhrig, New Journal of Physics 12, 033048 (2010).

43 M. Daghofer and M. Hohenadler, Phys. Rev. B 89, 035103 (2014).

44 T. Đurić, N. Chancellor, and I. F. Herbut, Phys. Rev. B 89, 165123 (2014). 